\title{
Peritoneum and Retroperitoneum
}

National Cancer Institute

\section{Source}

National Cancer Institute. Peritoneum and Retroperitoneum. NCI Thesaurus. Code C156712.

A term that refers to the peritoneum and the space behind it (retroperitoneum). 